Психология. Журнал Высшей школы экономики.

2018. T. 15. № 1. C. 128-144. DOI: 10.17323/1813-8918-2018-1-128-144

\title{
КОГНИТИВНАЯ ФЛЕКСИБИЛЬНОСТЬ ЛИЧНОСТИ: ТЕОРИЯ, ИЗМЕРЕНИЕ, ПРАКТИКА
}

\author{
Е.Ю. ОСАВОЛЮК ${ }^{a}$, С.С. КУРГИНЯН
}

${ }^{a}$ Национальный исследовательский университет «Высиая икола экономики», 1010о0, Россия, Москва, ул. Мясницкая, д. 20

\begin{abstract}
Резюме
Флексибильность рассматривается как специфическая способность личности, позволяющая ей организовывать свою познавательную деятельность и интеллектуальное поведение в зависимости от изменившихся условий. Несмотря на разработанность данного конструкта в разных контекстах его проявления, до сих пор в когнитивных исследованиях отсутствует его общее операциональное определение. Целью настоящего исследования является изучение существующих теоретических взглядов на проблему когнитивной флексибильности личности и определение их вклада в разработку способов ее измерения в практике оказания психологической помощи. В кратком обзоре отечественных и зарубежных когнитивных исследований обобщены представления о когнитивной флексибильности как ментальной способности, характеризующей умение индивида преобразовывать когнитивные установки в ответ на изменяющиеся условия его жизнедеятельности. Показано, что суцествует дефицит русскоязычных терминов, охватывающих описание исследуемого феномена, кроме того, он не находит должного рассмотрения относительно содержания и форм проявления в поведении человека, главным образом интеллектуального поведения. В зарубежных когнитивных исследованиях понятие «флексибильность» имеет широкое распространение. К нему обращаются при изучении познавательных способностей или умений. Для его операционального определения исследователи в большинстве дефиниций исходят из понимания флексибильности как существенного свойства когнитивной системы личности. Однако, чем собственно является это свойство, не раскрыто. В рамках настоящего обзора предложен подход к изучению флексибильности личности как специфической способности когнитивной системы и свойства различных познавательных процессов. При таком подходе способности определяются как свойства функциональной системы, реализующей конкретные психические функции. В ходе обзора процедур и методов измерения когнитивной флексибильности показано, что ввиду отсутствия операционального определения данного конструкта выбор инструментария, как правило, зависит от целей и задач исследования. Рассмотрены два вида основного инструментария, к которому обращаются исследователи: когнитивные пробы и опросники. Показана их пригодность в условиях оказания психологической помощи с целью проведения диагностики уровня выраженности когнитивной флексибильности, необходимого для перехода к адаптивному поведению.
\end{abstract}

Ключевые слова: интеллектуальное поведение, когнитивная флексибильность, ментальная способность, неадаптивные мысли, психическая ригидность.

Публикация подготовлена при поддержке Российского фонда фундаментальных исследований (проект № 17-06-00917). 
В психологии традиционно обращение к понятию «флексибильность» (англ. flexibility) связано с изучением познавательной деятельности и интеллектуального поведения человека (Ionescu, 2012). Широко используемые исследователями дефиниции данного понятия содержат такие наиболее существенные признаки, как гибкость, подвижность и приспособляемость. Зачастую именно отдельные признаки флексибильности рассматриваются в качестве конструктов, которыми описываются и объясняются когнитивные процессы и поведенческие проявления личности, характеризующие ее способность организовывать познавательную деятельность и интеллектуальное поведение в зависимости от изменившихся условий. С одной стороны, это приводит исследователей к неоднозначности в понимании сущности данного явления и ограниченности подходов в его изучении, с другой стороны, обращаясь к исследованию флексибильности, ученые, как правило, определяют ее в перспективе уровня рассмотрения данного явления в структуре личности. Отсюда ключевой проблемой при анализе понятия «флексибильность» является множественность определений.

При всей многочисленности определений данного понятия в отечественной психологии оно достаточно редко встречается. Обращение к нему главным образом связано с изучением противоположного конструкта - «ригидность». Так, например, в концепции Г.В. Залевского (2007) о фиксированных формах поведения флексибильность определяется в качестве противоположного психической ригидности полюса в континууме предиспозиционного фактора феноменологии фиксированных форм поведения человека. При этом автор рассматривает психическую ригидность как сложное многомерное свойство личности (или состояние), сочетающее в себе содержательную и формально-динамическую стороны, как трудность коррекции программы поведения в целом или ее отдельных элементов в связи с объективной необходимостью и разной степенью осознания и принятия этой необходимости индивидом (индивидуальная ригидность) и группой (групповая ригидность) и указывает на то, что психическая ригидность проявляет себя снижением адаптивных возможностей личности в связи с нарушением уровневых отношений в структуре действия.

Обзор отечественных психологических исследований последних десятилетий (20-30 лет) показал, что понятие «флексибильность» не только до настоящего времени не раскрыто в своем содержании, но и не предложены альтернативные концепции Г.В. Залевского подходы к его исследованию. В ряде работ, отражающих анализ особенностей организации когнитивной сферы личности и ее интеллектуального поведения, можно встретить обращение к конструкту «гибкость». Так, М.А. Холодная (2004), раскрывая концепцию когнитивных стилей и природы индивидуального ума, описывает один из стилей, который составляет основу феноменологии стилевого подхода, - «ригидный/ гибкий познавательный контроль». Данный когнитивный стиль, по 
определению автора, характеризует степень субъективной трудности в смене способов переработки информации в ситуации когнитивного конфликта и является одним из стилевых полюсов познавательного контроля. Заметим, что М.А. Холодная вводит стиль «ригидный/гибкий познавательный контроль», обращаясь к теории когнитивных контролей P. Гарднера с соавт. (Gardner et al., $1959,1960)$. В англоязычном оригинале текста исследований авторов теории данный вид контроля представлен как «constricted-flexible control». Мы видим, что один из его полюсов обозначен определением «flexible», который в переводе с английского означает «гибкий, податливый, легко приспосабливающийся» (Никошкова, 2006). Таким образом, можно констатировать, что при переводе англоязычного термина, обозначающего когнитивный стиль, М.А. Холодная, исходя из определенных концептуальных предположений, за основу взяла один из его смысловых эквивалентов. В данном случае в виде конструкта «гибкость». Здесь мы не ставим под сомнение адекватность перевода при введении новой терминологии в психологический категориальный аппарат. Нам важно показать существующее методологическое затруднение, с которым сталкивается исследователь при дефиците русскоязычных терминов, охватывающих описание исследуемого феномена.

Итак, для отечественной психологии неоднозначность в понимании сущности флексибильности личности и ограниченность подходов в изучении данного понятия обусловлены двумя, на наш взгляд, затрудне- ниями методологического плана. Во-первых, флексибильность рассматривается как противоположный ригидности конструкт и не находит должного рассмотрения относительно содержания и форм проявления в поведении человека (главным образом интеллектуального поведения). Во-вторых, в большинстве исследований англоязычный термин «flexibility», не имея транслитерации, получает интерпретацию одного из его смысловых эквивалентов - «гибкость», в то время как понятие «rigidity» имеет транслитерированный перевод в русском языке и обозначается термином «ригидность».

В зарубежных исследованиях понятие «флексибильность» широко распространено в когнитивных исследованиях. К нему, как правило, обращаются при изучении познавательных способностей или умений. Отсюда данное понятие в англоязычной терминологии обозначается как «когнитивная флексибильность» (КФ). Теа Ионеску (Ionescu, 2012) в своем теоретическом обзоре зарубежной литературы за последние 100 лет пришла к выводу, что понятие «флексибильность» достаточно сложно определить, поскольку оно является интуитивным, в то время как конструкт «когнитивная флексибильность» обнаруживается в широком спектре форм поведения человека. В ходе анализа современных психологических исследований ею выделены когнитивный и личностный уровни изучения КФ, на которых данный конструкт рассматривается как свойство когнитивной системы, основанное на взаимодействии различных механизмов познания, актуализирующихся в ответ на требования 
среды для достижения флексибильного поведения. На когнитивном уровне происходит взаимодействие таких механизмов познания, как, например, исполнительные функции, внимание, репрезентации, восприятия, согласование параметров задачи с целями, мониторинг конфликтов, предшествующих знанию, на личностном уровне - взаимодействие сенсомоторных механизмов с механизмами познания и условиями развития конкретного индивида. Предложенный Ионеску комплексный подход к исследованию КФ как свойства когнитивной системы позволяет рассматривать понятие «флексибильность» в разных контекстах его проявления.

В настояшем исследовании мы ставим целью изучение существуюших теоретических взглядов на проблему когнитивной флексибильности личности и определение их вклада в разработку способов ее измерения в практике оказания психологической помощи.

\section{Флексибильность личности и ее изучение в когнитивных исследованиях}

Множественность определений понятия «флексибильность» является следствием разработки различных подходов к изучению соотносимых с ним феноменов, а отсутствие его общего операционального определения приводит к ограниченности этих подходов (Ionescu, 2012). В зависимости от уровня рассмотрения данного явления в структуре личности выделяются основания для его операционального определения. Исследователи в большинстве дефиниций исходят из понимания флексибильности как специфической способности или механизма когнитивной системы, а также как свойства различных когнитивных процессов. При этом здесь мы ограничимся обзором тех исследований, предметом которых является когнитивная флексибильность, и затронем те области науки, для которых познавательная деятельность и интеллектуальное поведение человека составляют объект исследования.

Как специфическая способность КФ содержательно определяется через конструкт «переключаемость» (shifting). Данный конструкт в большинстве исследований синонимичен флексибильности. Им обьясняется способность индивида быстро переключаться при решении задач с одного критерия (правила) на другой (Cragg, Chevalier, 2006; Colzato et al., 2009). Несомненно, что при таком подходе КФ приобретает конкретное значение, однако при анализе флексибильного поведения можно обнаружить, что «переключаемость» будет одной из его характеристик (Ionescu, 2012). Исследователи, которые определяют КФ свойством различных когнитивных процессов, как правило, обращаются к анализу внешних факторов (например, параметры задания, опыт и др.), способствующих проявлению флексибильного поведения (Blaye, Bonthoux, 2001; Deak, 2003; Plunkett, 2006). В подобных исследованиях изучаемый процесс, например категоризации (Ross, Murphy, 1999), сводится к анализу меры выраженности флексибильности как свойства этого процесса. При данном подходе наблюдается дефицит содержательного понимания 
КФ как свойства когнитивных процессов. Здесь очевидно операциональное определение свойства процесса в той степени, в которой оно измеряется, однако остается непонятным, что содержательно представляет собой это свойство.

Современные исследования КФ затрагивают такие области научного знания, как психология образования и когнитивная нейронаука. Весомый вклад в исследование данного конструкта привнесла психология образования, в рамках которой была разработана теория когнитивной флексибильности. Ее разработчиками являются Спиро и Дженг (Spiro, Jehng, 1990). Предложенная ими модель обращена к изучению процессов обучения с недостаточно структурированной информацией для ее освоения. По мнению авторов, процессы такого рода составляют реальную основу современной жизни индивида. Отсюда под КФ авторы понимают способность человека к спонтанной реструктуризации собственных знаний, которая в большинстве случаев проявляется как приспособительная (адаптивная) реакция на существенные изменения, происходящие во внешней среде. В когнитивной нейронауке исследование КФ осуществляется во взаимосвязи с изучением высших психических функций и их проявлений в областях головного мозга, а именно в лобных долях (Stuss, Alexander, 2000), базальных ганглиях, передней поясной и префронтальной коре, затылочной и теменной коре (Leber et al., 2008). Фокус внимания исследователей направлен на процесс активизации конкретных мозговых структур в рамках выпол- нения определенной деятельности с изменяющимися условиями. На уровне содержательного анализа данного явления в поведении индивида предложены такие его определения, как адаптивная способность индивида, связанная с приобретением опыта, освоением стратегий познания и модификацией традиционного способа решения задачи (Canas et al., 2003); способность индивида изменять мысли и ход действий в своем поведении в ответ на изменение ситуации (Canas et al., 2005; Lezak, 2004); способность адаптироваться в измененных условиях окружающей среды и генерировать новые идеи (Barbey el al., 2013). КФ считается одним из маркеров адаптивного поведения, проявляющимся в умении ставить перед собой новые цели в соответствии с изменениями внешних условий и действовать согласно им (Yehene et al., 2008).

Особый интерес к данному явлению проявляют исследователи интеллектуальных способностей, креативности и особенностей организации когнитивной сферы личности. Так, в работах Дж. Гилфорда флексибильность рассматривалась как способность разрывать устоявшиеся когнитивные паттерны мышления, создавая новые (творческие) ассоциации между понятиями (Guilford, 1967). В исследованиях, отражающих анализ творческой деятельности в повседневной жизни индивида, КФ признается важной составной частью креативности (Runco, 2004), ее ядром и необходимым компонентом (Baghetto, Kaufman, 2007; Hennessey, Amabile, 2010). На уровне организации когнитивной сферы личности КФ выступает ее динамическим 
свойством. Динамизм проявляется во взаимодействии механизмов познания на разных уровнях функционирования личности: когнитивном и личностном (Ionescu, 2012). Здесь исходным основанием для понимания флексибильного поведения индивида являются динамическая активизация и преобразование когнитивных процессов в ответ на изменяюшиеся условия (Deak, 2003). В свою очередь, это преобразование приводит к тому, что индивид иначе воспринимает ситуацию, у него появляются альтернативные способы реагирования на нее (Johnco et al., 2014a). Подобное объяснение является свидетельством проявления КФ как способности генерировать несколько путей решения проблемы, переключаться между различными классами знаний и снижать уровень привычных реагирований в пользу альтернативных ответов (Rende, 2000), как способности давать возможные объяснения переживаемым жизненным событиям, трудным ситуациям и предлагать различные варианты выхода из них (Dennis, Vander Wal, 2010).

Проведенный обзор существующих подходов к изучению флексибильности личности показал, что, несмотря на разработанность данного конструкта в когнитивных исследованиях, они ограничены отсутствием системного взгляда на его операциональное определение. Главным образом наблюдается дефицит в интегративном подходе, который позволил бы изучать конструкт «когнитивная флексибильность» в системе его основных определений, а именно как специфическую способность или механизм когнитивной системы и как свойство различных когнитивных процессов. В этом вопросе мы согласны с Ионеску: существует достаточно много различных подходов к изучению соотносимых с КФ феноменов, однако отсутствует единый подход к пониманию данного явления (Ionescu, 2012). Все же нам представляется, что, сделав свой основной акцент в предложенной ею теоретической модели КФ на понимании данного конструкта как динамического свойства когнитивной системы, Ионеску ограничилась определением его как существенного признака системы и описанием его роли в ней. И все же, чем собственно является это свойство, не раскрыто. Мы, в свою очередь, полагаем, что КФ может рассматриваться в качестве специфической способности, характеризующейся функционированием когнитивной системы личности, и свойства различных когнитивных процессов. Здесь мы основываемся на понимании способностей, предложенном В.Д. Шадриковым в рамках его теории: «Способность выступает конкретным проявлением психической функции (восприятия, памяти, мышления...), психическая функция реализуется определенной функциональной системой, механизм психического процесса описывается в системе физиологических понятий, характеризующих деятельность функциональной системы, реализующей определенную психическую функцию, конечные характеристики процесса описываются в терминах свойств и отношений внешних объектов» (Шадриков, 2007, с. 45). В рамках данного подхода способности и познавательные процессы являются проявлением 
функциональных физиологических систем, реализующих конкретные психические функции. При этом характеристики способностей дополняются свойствами и качествами результатов познавательных процесcoB.

Таким образом, наше определение когнитивной флексибильности личности в широком смысле основывается на понимания данного явления как способности, которая проявляется на уровне ее когнитивного функционирования. В узком смысле КФ - это ментальная способность, характеризующая умение индивида преобразовывать когнитивные установки в ответ на изменяющиеся условия его жизнедеятельности (Кургинян, Осаволюк, 2018).

\section{Измерение когнитивной флексибильности}

В настоящее время существует большое количество методик, к которым обращаются исследователи при изучении КФ. Однако ввиду отсутствия операционального определения данного конструкта выбор инструментария, как правило, зависит от целей и задач исследования. Выделяют две группы методик измерения КФ: когнитивные пробы и самоотчет.

Первую группу составляют инструменты, предназначенные для измерения характеристик КФ, проявляющих себя в когнитивных процессах. Оценка степени затруднения испытуемого при изменении способа переработки информации в ситуации когнитивного конфликта методикой «Словесно-цветовая интерференция» Дж.Р. Струпа (Stroop Color and Word Test) позволяет установить меру выраженности его способности справляться с когнитивным стрессом и обрабатывать сложные данные (Golden, 1978; Golden, Golden, 2002). В нейропсихологических исследованиях способность индивида к произвольному преобразованию линии поведения обозначается термином «переключаемость». Переключаемость выступает в роли компонента исполнительного функционирования (ехеcutive functions) (Алексеев, Рупчев, 2010) и измеряется методикой «Тест связи чисел» (Trail Making Test). Успешное выполнение теста связано c соединением последовательности чисел (Reitan, Wolfson, 1993). Оценка переключаемости в условиях изменения подкрепления (Heaton, PAR Staff, 2003) осуществляется методикой «Висконсинский тест сортировки карточек» (Wisconsin Card Sorting Test). Данный инструмент позволяет через анализ когнитивных функций оценить способность индивида к выделению абстрактных категорий и концентрации внимания (Полунина, Давыдов, 2004). Измерение КФ осуществляется также через комплексную оценку когнитивных функций (память, внимание, речь, речевая активность и зрительно-пространственные функции) при помощи методики «Адденбрукская когнитивная шкала» (Addenbrooke's Cognitive Examination-Revised; Mioshi et al., 2006). Вербальная и невербальная беглость как характеристики речевого компонента когнитивных функций измеряются методиками Controlled Oral Word Associations Test (Benton, Hamsher, 1976) и Ruff Figural Fluency Test (RFFT; Ruff, 1996). 
Вторую группу составляют методики, основанные на самоотчете. В настоящее время они получают широкое распространение за счет простой процедуры регистрации данных и получения быстрого диагностического результата (Dennis, Vander Wal, 2010). Данный инструментарий направлен на оценку личностных особенностей индивида на уровне анализа организации его когнитивной сферы и интеллектуального поведения. Здесь следует отметить, что первоначально не все существующие методики самоотчета, используемые для измерения КФ, были целенаправленно разработаны для этого. Например, опросник атрибутивного стиля (Peterson et al., 1982) был разработан для определения атрибутивного стиля или склонности соотносить определенные причины и следствия при объяснении событий. Респондентам необходимо описать свои чувства по отношению к гипотетическим ситуациям (позитивным или негативным), связанные с их возникновением, а затем при помощи шкалы Лайкерта ответить на вопросы о причинах возникновения указанной ситуации. КФ в данном случае оценивается через измерение когнитивной ригидности, которая проявляется в виде тенденции испытуемого указывать максимальные или минимальные значения по шкале (Teasdale et al., 2001). Таким образом, при помощи данного инструмента КФ опосредованно оценивается через конструкт «ригидность» в гипотетических ситуациях, однако данный аспект может накладывать ограничения при работе с уже совершившимися событиями, имеющими значение для текущего состояния испытуемого.
В настоящее время существуют два инструментария оценки КФ, основанные на самоотчете: «Шкала когнитивной флексибильности» (Cognitive Flexibility Scale (CFS); Martin, Rubin, 1995) и «Опросник когнитивной флексибильности» (Cognitive Flexibility Inventory; Dennis, Vander Wal, 2010). Каждый из них разработан для измерения различных аспектов КФ. Так, «Шкала когнитивной флексибильности» представляет собой опросник из 12 пунктов, содержание которых включает три аспекта измерения КФ: (1) осознание альтернатив в общении, (2) готовность адаптироваться к сложившейся ситуации и (3) уверенность в собственной флексибильности. Данная методика направлена на оценку КФ индивида в установлении межличностных отношений и ее роли в формировании коммуникативной компетентности. Авторами высказано предположение о низкой степени подверженности коммуникативно компетентных лиц депрессии (Martin, Anderson, 1998). Исследования КФ в коммуникативной сфере во многом соотносятся с предположением авторов методики. В ряде исследований показано, что у индивидов с более конструктивным подходом к поиску выхода из проблемной ситуации КФ является важным компонентом коммуникативной компетенции (O'Keefe, Delia, 1982), а у индивидов с выраженной готовностью быть флексибильными КФ является проявлением влияния внутриличностных коммуникативных процессов на качество межличностного общения (Lippard-Justice, 1989). На уровне анализа коммуникативного поведения личности было 
установлено, что на проявление КФ влияет внутренняя мотивационная структура, определяющая готовность человека адаптироваться к ситуации для удовлетворения текущих потребностей (McCloskey, Cohen, 1989). Авторами-разработчиками были проведены дополнительные исследования, результаты которых выявили положительную связь КФ с такими личностными характеристиками, как уверенность в себе, отзывчивость, самооценка, отраженное отношение к себе (значимых других) и уверенность в своей активной коммуникативной позиции.

Опросник когнитивной флексибильности (Cognitive Flexibility Inventory (CFI); Dennis, Vander Wal, 2010) представляет собой краткий тест самоотчета. Авторы-разработчики стремились создать инструментарий, который позволил бы получить быстрый результат диагностики КФ и мог бы быть использован неоднократно на протяжении всего исследования или психотерапевтического воздействия. Методика разработана для измерения трех аспектов КФ: (1) склонности к восприятию сложных ситуаций как контролируемых; (2) способности к восприятию нескольких альтернативных объяснений жизненных проявлений и поведения людей; (3) способности к генерированию нескольких альтернативных вариантов решений в сложных ситуациях. По мнению авторов, выраженность данных аспектов в поведении индивида позволяет ему при встрече со стрессовыми жизненными событиями (ситуациями) преобразовывать свое поведение в ответ на изменившиеся условия. Основной целью при разработке методики было создать инструмент с несколькими надежными и валидными шкалами для измерения КФ. Методика состоит из 20 пунктов и включает две субшкалы: «Альтернативы» для оценивания способности человека давать альтернативные объяснения сложившейся ситуации и создавать новые различные решения; и «Контроль» для оценивания способности человека воспринимать сложные ситуации как контролируемые.

Итак, проведенный обзор инструментария для измерения КФ показал, что предназначение и направленность методик первой группы (когнитивные пробы) главным образом направляет исследователя к оценке когнитивных функций, процессов и свойств индивида, в то время как методики второй группы (опросники) - к оценке личностных характеристик индивида на уровне анализа организации его когнитивной сферы и интеллектуального поведения. Имеющийся инструментарий остается актуальным в нейрокогнитивных исследованиях и психологической практике. Однако при выборе методики измерения КФ исследователи сталкиваются с рядом ограничений в ее использовании. Как правило, они связаны с временни́ми затратами на проведение и обработку данных исследования (обследования), объемом регистрируемых данных и интерпретацией результатов, а также условиями взаимодействия испытуемого (обследуемого) и экспериментатора (диагноста). Когнитивные пробы в отличие от опросников требуют больше времени на их проведение и обработку результатов. Объем регистрируемых 
данных и их интерпретация накладывают ограничения на их использование в психотерапевтической практике на этапе диагностики выраженности КФ (Dennis, Vander Wal, 2010). В целом методики, основанные на самоотчете (CFS и CFI), имеют высокие показатели конвергентной валидности и низкие показатели в сравнении с методиками, предназначенными для измерения характеристик КФ (ACE-R, WCST, TMT, COWAT, SCWT, RFFT), проявляющих себя в когнитивных процессах (Johnco et al., 2014a).

\section{Диагностика когнитивной флексибильности в практике оказания психологической помощи}

Особый интерес для практикующих специалистов представляет использование результатов измерительных процедур и методик диагностики КФ в когнитивно-бихевиоральной терапии (КБТ). Корректная диагностика уровня выраженности КФ способствует продуктивной работе с неадаптивными мыслями клиентов (Dennis, Vander Wal, 2010). На этапе диагностического обследования в работе терапевта важным является не только определение индивидуально-психологических особенностей личности клиента, но и установление уровня выраженности у него КФ. Именно от того, насколько правильно терапевт смог определить наличный уровень КФ, во многом зависят выявление деструкции неадаптивных мыслей и последовательное развитие у клиента способности к адаптивному мышлению (Young et al., 2001).
Диагностика КФ на разных этапах психотерапевтического воздействия способствует правильному пониманию проявления отдельных форм фиксированного поведения клиента. В ряде исследований показано, что принятие неадаптивных мыслей связано с недостаточной выраженностью КФ в мыслительных процессах (Teasdale et al., 1995). Направленность психотерапии в этом случае связывают с развитием у клиента способности к преобразованию когнитивных установок в ответ на изменяющиеся условия его жизнедеятельности. Внимание исследователей к диагностическому этапу в процессе терапевтического лечения, как правило, обусловлено изучением условий выбора наиболее приемлемого метода, отдельных техник и в целом формы терапии (индивидуальная или групповая). Особый интерес представляют диагностика КФ в начале лечения и фиксация конечного результата по истечении курса лечения. Например, в обследовании лиц, склонных к перфекционизму (Nazarzadeh et al., 2015), было зафиксировано изменение у них диагностированного в начале групповой КБТ уровня КФ. Данный эффект обнаружен благодаря оценке способности пациентов осознавать возможности выбора стиля своего мышления. Отсюда правильно подобранные техники терапевтического воздействия с учетом установленного уровня КФ позволили к концу курса терапии снизить у них уровень неадаптивных мыслей, преодолеть привычные установки и закрепить тенденцию быть более когнитивно флексибильными в повседневной жизни. Следует также отметить, что 
продолжительность лечения во многом определяется наличным уровнем КФ, установленным на начальном этапе психотерапевтического воздействия. Как правило, учет уровня КФ, достаточного для перехода к адаптивному поведению, позволяет проводить дифференциацию клиентов при групповой форме КБТ.

В отдельных случаях техники терапевтического воздействия являются средством установления уровня КФ. При этом индикатором диагностированного уровня выступают характер и объем затруднений испытуемых, с которыми они сталкиваются в ходе работы с техникой, и их навыки находить разнообразные способы совладания с этими затруднениями. Так, у лиц с низким уровнем КФ затруднения возникают в ходе работы с техникой «когнитивное реструктурирование», что является свидетельством низкого чувства контроля над ситуацией при условии последовательной отработки алгоритма ее разрешения (Johnco et al., 2014b).

В настоящее время особый интеpec у практикующих специалистов вызывают методы диагностики КФ, основанные на самоотчете (Tokuyoshi, Iwsaki, 2012; Wang et al., 2016; Gülüm, Dag, 2012; Shareh et al., 2014). При работе с подобным инструментарием у терапевта появляется возможность оценки изменений когнитивных установок лиц, находящихся в процессе КБТ. Оценка сдвигов в уровне КФ позволяет проследить, насколько клиент способен изменять свое отношение к дисфункциональным мыслям и чувствам, не прибегая к осознанной модификации их содержания (Teasdale et al., 2001).
Денис и Вандер Вал (Dennis, Vander Wal, 2010) в практических целях считают самоотчет более удобным инструментарием по сравнению с измерениями, основанными на анализе поведенческих проявлений индивида, поскольку он менее трудоемкий и простой в использовании и подсчете баллов.

\section{Заключение}

На данный момент в психологии и смежных с ней науках наблюдается большой интерес исследователей к когнитивной флексибильности личности. Являясь одной из ключевых характеристик познавательной деятельности и интеллектуального поведения, данный конструкт не нашел своего должного рассмотрения в работах отечественных психологов. В настоящей работе проведен обзор существующих подходов к разработке его операционального определения, процедур и методов измерения, а также диагностике в практике оказания психологической помоши. Определение КФ базируется на анализе когнитивной сферы личности (познавательных процессов и их свойств) и ее функционирования (познавательной деятельности и интеллектуального поведения). КФ рассматривается как ментальная способность, характеризующая умение индивида преобразовывать когнитивные установки в ответ на изменяющиеся условия его жизнедеятельности. Такой подход к определению КФ представляется перспективным с точки зрения операционализации данного понятия, поскольку он позволяет не только выделить КФ в ряду соотносимых 
понятий, но и определить ее как способность личности, которая проявляется на уровне ее когнитивного функционирования. Существующие методы измерения КФ (когнитивные пробы и опросники) ограничивают исследователя в выборе, главным образом это касается временни́х затрат на проведение и обработку данных исследования (обследования), а также их использования в психотерапевтической практике. Эффективность организации терапевтического воздействия и выбора наиболее приемлемой техники в каждом конкретном случае зависит от того, насколько правильно диагностирован наличный уровень КФ в начале курса лечения. Учет уровня КФ, необходимого для перехода к адаптивному поведению, способствует продуктивной работе с неадаптивными мыслями клиентов в когнитивно-бихевиоральной терапии.

Дальнейшее рассмотрение изучаемого конструкта требует перехода от его концептуального осмысления к эмпирическому исследованию. Для этого нами проведена апробация Опросника когнитивной флексибильности на русскоязычной выборке (CFI-R) и предложена его русскоязычная версия (Кургинян, Осаволюк, 2018). Особый интерес для нас в перспективе дальнейших исследований представляет изучение КФ личности в ее отношении к себе. Здесь оценка вклада уровня выраженности КФ индивида наряду с его личностными характеристиками в процесс обращения с собой позволит не только проводить анализ его когнитивного функционирования, но и прогнозировать его интеллектуальное поведение.

\section{Литература}

Алексеев, А. А., \& Рупчев, Г. Е. (2010). Понятие об исполнительных функциях в психологических исследованиях: перспективы и противоречия. Психологиеские исследования: электронный науиный журиал, 4, 6. Режим доступа: http://psystudy.ru

Залевский, Г. В. (2007). Лииность и фиксированные формы поведения. М.: Изд-во «Институт психологии РАН».

Кургинян, С. С., Осаволюк, Е. Ю. (2018). Опросник когнитивной флексибильности: структура русскоязычной версии (CFI-R), надежность и валидность опросника. Психологический журнал, 39(2), 105-119.

Никошкова, Е. В. (2006). Англо-русский словарь по психологии. М.: РУССО.

Полунина, А. Г., Давыдов, Д. М. (2004). Висконсинский тест сортировки карточек как инструмент оценки когнитивных функций. В кн. Т. Б. Дмитриева (ред.), Социальная психиатрия (c. 217-236). М.: Изд-во ГНЦ ССП им. В.П. Сербского.

Холодная, М. А. (2004). Когнитивиые стили. О природе индивидуального ума. СПб.: Питер.

Шадриков, В. Д. (2007). Ментальное развитие иеловека. М.: Аспект Пресс.

Ссылки на зарубежные истоиники см. в разделе References после англоязыного блока. 
Осаволюк Екатерина Юрьевна - аспирант, департамент психологии, факультет социальных наук; стажер-исследователь, научно-учебная лаборатория психологии способностей, Национальный исследовательский университет «Высшая школа экономики».

Сфера научных интересов: флексибильность личности, отношение к себе.

Контакты: eosavoluk@hse.ru

Кургинян Сергей Сергеевич - заведующий лабораторией, Научно-учебная лаборатория психологии способностей; доцент, департамент психологии, факультет социалыных наук, Национальный исследовательский университет «Высшая школа экономики», кандидат психологических наук.

Сфера научных интересов: адаптивно-компенсаторные структуры, психические ресурсы, отношение к себе.

Контакты: skurginyan@hse.ru

\title{
Person's Cognitive Flexibility: Theory, Measurement, and Practice
}

\author{
E.Yu. Osavolyuk ${ }^{a}$, S.S. Kurginyan ${ }^{a}$
}

${ }^{a}$ National Research University Higher School of Economics, 20 Myasnitskaya str., Moscow, 101000, Russian Federation

\begin{abstract}
Cognitive flexibility is considered as a person's mental ability to organize one's own cognitive activity and intellectual behavior in response to changing environmental conditions. Though the construct of cognitive flexibility has been well investigated in different contexts of its manifestation, yet there is no general operational definition of this construct in cognitive studies. This study aimed to explore the current theoretical views on the problem of a person's cognitive flexibility and to indicate the contribution of these views to the development of the measures of cognitive flexibility within the psychological treatment. In brief review of Russian and foreign cognitive studies, the ideas about the term "cognitive flexibility" has been summarized as a mental ability of a person, a skill to change cognitive attitudes in response to the changing conditions of one's own life. It was shown that there has been a lack of definitions in Russian to describe the phenomenon of cognitive flexibility, and it has not been studied in terms of content and forms of its manifestation in human behavior, especially, in intellectual behavior. In foreign cognitive studies the term cognitive flexibility is widespread. It is referred to in studies of cognitive abilities and skills. The majority of researchers determine its operational definition as a general property of personality's cognitive system. At the same time, it is not known what this property is. For the purposes of the present study, the approach to examine the person's flexibility has been put forward. It was considered as a specific ability of cognitive system and a property of different cognitive processes. This approach uses abilities as the properties of functional system, which bring about specific mental functions. In reviewing the existing measures of cognitive flexibility, it was demonstrated that the lack of operational definition of this construct usually leads to the choice of the instrument in accordance to aims and goals of the research. Cognitive tests and self-report measures were considered as two main instruments in examining person's flexibility. Their appli-
\end{abstract}


cability was proved and it was shown that the instruments examine the type of cognitive flexibility needed for change to adaptive behavior within psychological treatment.

Keywords: intellectual behavior, cognitive flexibility, mental ability, maladaptive thoughts, mental rigidity.

\section{References}

Alekseev, A. A., \& Rupchev, G. E. (2010). Ponjatie ob ispolnitel'nyh funkcijah v psihologicheskih issledovanijah: perspektivy i protivorechija [The notion of executive functions in psychological studies: perspectives and contradictions]. Psikhologicheskie issledovaniya, 4, 6. Retrieved from http://psystudy.ru

Baghetto, R., \& Kaufman, J. (2007). Toward a broader conception of creativity: A case for "mini-c" creativity. Psychology of Aesthetics, Creativity, and the Arts, 1, 73-79.

Barbey, A. K., Colom, R., \& Grafman, J. (2013). Architecture of cognitive flexibility revealed by lesion mapping. Neurolmage, $82,547-554$.

Benton, A. L. \& Hamsher, K. (1976). Multilingual aphasia examination. Iowa City, IA: University of Iowa.

Blaye, A., \& Bonthoux, F. (2001). Thematic and taxonomic relations in preschoolers: the development of flexibility in categorization choices. British Journal of Developmental Psychology, 19, 395-412.

Canas, J. J., Antoli, A., Fajardo, I., \& Salmeron, L. (2005). Cognitive inflexibility and the development and use of strategies for solving complex dynamic problems: Effects of different types of training. Theoretical Issues in Ergonomics Science, 6(1), 95-108.

Canas, J. J., Quesada, J. F., Antolí, A., \& Fajardo, I. (2003). Cognitive flexibility and adaptability to environmental changes in dynamic complex problem-solving tasks. Ergonomics, 46(5), 482-501.

Colzato, L. S., Huizinga, M., \& Hommel, B. (2009). Recreational cocaine polydrug use impairs cognitive flexibility but not working memory. Psychopharmacology, 207, 225-234.

Cragg, L., \& Chevalier, N. (2006). The processes underlying flexibility in childhood. The Quarterly Joumal of Experimental Psychology, 65(2), 209-320.

Deak, G. O. (2003). The development of cognitive flexibility and language abilities. Advances in Child Development and Behavior, 31, 271-327.

Dennis, J. P., \& Vander Wal, J. S. (2010). The cognitive flexibility inventory: Instrument development and estimates of reliability and validity. Cognitive Therapy and Research, 34(3), 241-253.

Gardner, R. W., Holzman, P. S., Klein, G. S., Linton, H. B., \& Spence, D. P. (1959). Cognitive control. A study of individual consistencies in cognitive behavior. Psychological Issues, 4(1), 1-186.

Gardner, R.W., Jackson, D.N., \& Messick, S.J. (1960). Personality organization in cognitive controls and intellectual abilities. Psychological Issues, 8(2), 149.

Golden, C. J. (1978). Stroop Color and Word Test: A manual for clinical and experimental uses. Wood Dale, IL: Stoelting Co.

Golden, Z., \& Golden, C. J. (2002). Patterns of performance on the Stroop Color and Word Test in children with learning, attentional, and psychiatric disabilities. Psychology in the Schools, 39(5), 489-495.

Guilford, J. P. (1967). The nature of human intelligence. New York: McGraw-Hill Book Company. 
Gülüm, İV., \& Dag, I. (2012). The Turkish adaptation, validity and reliability study of the Repetitive Thinking Questionnaire and the Cognitive Flexibility Inventory. Anatolian Joumal of Psychiatry, 13, 216-223. (in Turkish, abstract in English)

Heaton, R. K., \& PAR Staff. (2003). Wisconsin Card Sorting Test: Computer version 4. Odessa, FL: Psychological Assessment Resources.

Hennessey, B. A., \& Amabile, T. M. (2010). Creativity. Annual Review of Psychology, 569-598.

Ionescu, Th. (2012). Exploring the nature of cognitive flexibility. Ner Ideas in Psychology, 30(2), 190-200.

Johnco, C., Wuthrich, V. M., \& Rapee, R. M. (2014a). Reliability and validity of two self-report measures of cognitive flexibility. Psychological Assessment American Psychological Association, 26, 1381-1387.

Johnco, C., Wuthrich V. M., \& Rapee R. M. (2014b). The influence of cognitive flexibility on treatment outcome and cognitive restructuring skill acquisition during cognitive behavioral treatment for anxiety and depression in older adults: results of a pilot study. Behaviour Research and Therapy, 57, 55-64.

Kholodnaya, M. A. (2004). Kognitiomye stili. O prirode individual'nogo uma [Cognitive styles. On the nature of individual mind]. Saint Petersburg: Piter.

Kurginyan, S. S., \& Osavolyuk, E. Yu. (2018). Oprosnik kognitivnoj fleksibil'nosti (CFI): adaptacija na russkojazychnoj vyborke [The cognitive flexibility inventory (CFI): adaptation for Russian-speaking sampling]. Psikhologicheskii Zhumal, 39(2), 105-119.

Leber, A. B., Turk-Browne, N. B. \& Chun, M. M. (2008). Neural predictors of moment-to-moment fluctuations in cognitive flexibility. Proceedings of the National Academy of Sciences of the United States of America, 105(36), 13592-13597. doi:10.1073/pnas.0805423105

Lezak, M. D., Howieson, D. B., Loring, D. W., Hannay, H. J, \& Fischer, J. S. (2004). Neuropsychological assessment. New York: Oxford University Press.

Lippard-Justice, P. (1989). The relationship between intrapersonal and interpersonal communication patterns. In C. V. Roberts, K. Watson, \& L. L. Barker (Eds.), Intrapersonal communication processes: Original essays (pp. 444-455). New Orleans: SPECTRA.

Martin, M. M., \& Anderson, C. M. (1998). The cognitive flexibility scale: Three validity studies. Communication Reports, 11(1), 1-9.

Martin, M. M., \& Rubin, R. B. (1995). A new measure of cognitive flexibility. Psychological Reports, 76, 623-626.

McCloskey, M., \& Cohen, N. J. (1989). Catastrophic interference in connectionist networks: The sequential learning problem. Psychology of Learning and Motivation, 24, 109-165.

Mioshi, E., Dawson, K., Mitchell, J., Arnold, R., \& Hodges, J. R. (2006). The Addenbrooke's Cognitive Examination Revised (ACE-R): a brief cognitive test battery for dementia screening. Intermational Journal of Geriatric Psychiatry, 21, 1078-1085. doi:10.1002/gps.1610

Nazarzadeh, R. S., Fazeli, M., Aval, M. M., \& Shourche, R. M. (2015). Effectiveness of cognitive-behavior therapy on cognitive flexibility in perfectionist. Psychology, 6, 1780-1785.

Nikoshkova E.V. (2006). Anglo-russkiy slovar' po psikhologii [English Russian Dictionary of Psychology]. Moscow: RUSSO.

O'Keefe, B. J., \& Delia, J. G. (1982). Impression formation and message production. In M. E. Roloff \& C. R. Berger (Eds.), Social cognition and communication (pp. 33-72). Beverly Hills, CA: Sage.

Peterson, C., Semmel, A., von Bayer, C., Abramson, L., Metalsky G., \& Seligman, M. E. P. (1982). The attributional style questionnaire. Cognitive Therapy and Research, 6(3), 287-300. 
Plunkett, K. (2006). Learning how to be flexible with words. In Y. Munakata, \& M. H. Johnson (Eds.), Attention and performance XXI: Processes of change in brain and cognitive development (pp. 233-248). New York: Oxford University Press.

Polunina, A. G., \& Davydov, D. M. (2004). Viskonsinskij test sortirovki kartochek kak instrument ocenki kognitivnyh funkcij [Wisconsin Card Sorting Test as a tool for assessment of cognitive functions]. In T. B. Dmitrieva (Ed.), Social'naja psihiatrija [Social Psychiatry] (pp. 217-236). M.: Izdatel'stvo GNC SSP by V.P. Serbskii.

Reitan, R. M., \& Wolfson, D. (1993). The Halstead-Reitan Neuropsychological Test Battery: Theory and clinical interpretation (2nd ed.). Tucson, AZ: Neuropsychological Press.

Rende, B. (2000) Cognitive flexibility: Theory, assessment, and treatment. Seminars in Speech and Language, 21(2), 121-133.

Ross, B. H., \& Murphy, G. L. (1999). Food for thought: cross-classification and category organization in a complex real-world domain. Cognitive Psychology, 38, 495-553.

Ruff, R. M. (1996). Ruff figural fluency test. Test. Professional manual. Odessa, FL: Psychological Assessment Resources.

Runco, M. A. (2004). Creativity. Annual Review Psychology, 55, 657-687.

Shadrikov, V. D. (2007). Mental'noe razvitie cheloveka [Human mental development]. Moscow: Aspekt Press.

Shareh, H., Farmani, A., \& Soltani, E. (2014). Investigating the reliability and validity of the cognitive flexibility inventory (CFI-I) among Iranian university students. Practice in Clinical Psychology, $2(1), 43-50$.

Spiro, R. J. \& Jehng, J. C. (1990). Cognitive flexibility and hypertext: Theory and technology for the nonlinear and multidimensional traversal of complex subject matter. In D. Nix \& R. Spiro (Eds.), Cognition, education, and multimedia: Exploring ideas in high technology (pp. 163-205). Hillsdale, NJ: Lawrence Erlbaum Associates.

Stuss, D. T., \& Alexander, M. P. (2000). Executive functions and the frontal lobes: A conceptual view. Psychological Research, 63(3-4), 289-298.

Teasdale, J. D., Scott, J., Moore, R. G., Hayhurst, H., Pope, M., \& Paykel, E. S. (2001). How does cognitive therapy prevent relapse in residual depression? Evidence from a controlled trial.Joumal of Consulting and Clinical Psychology, 69, 347-357.

Teasdale, J. D., Segal, Z., \& Williams, J. M. (1995). How does cognitive therapy prevent depressive relapse and why should attentional control (mindfulness) training help? Behaviour Research and Therapy, 33(1), 25-39.

Tokuyoshi, Y., \& Iwsaki, S. (2012). Development and validation of cognitive flexibility inventory Japanese (in Japanese). Proceedings of the 76th Anmual Convention of the Japanese Psychological Association. Tokyo (p. 672).

Wang, Y., Yang, Y., Xiao, W., \& Su, Q. (2016). Validity and reliability of the Chinese version of the Cognitive Flexibility Inventory in college students. Chinese Mental Health Journal, 1, 58-63.

Yehene, E., Meiran, N., \& Soroker, N. (2008). Basal ganglia play a unique role in task switching within the frontal-subcortical circuits: evidence from patients with focal lesions. Journal of Cognitive Neuroscience, 20(6), 1079-1093.

Young, J. E., Weinberger, A. D., \& Beck, A. T. (2001). Cognitive therapy for depression. In D. H. Barlow (Ed.), Clinical handbook of psychological disorders: A step-by-step treatment manual (3rd ed., pp. 264-308). New York: Guilford Press. 
Zalevsky, G. V. (2007). Lichnost'i fiksirovannye formy povedeniya [Personality and fixed forms of behavior]. Moscow: Institute of Psychology of RAS.

Ekaterina Yu. Osavolyuk - Ph.D. student, School of Psychology, Faculty of Social Sciences; research assistant, Scientific-Educational Laboratory of Ability Psychology, School of Psychology, Faculty of Social Sciences, National Research University Higher School of Economics.

Research area: person's flexibility, self-relation.

E-mail: eosavoluk@hse.ru

Sergey S. Kurginyan - head, Scientific-Educational Laboratory of Ability Psychology; associate professor, School of Psychology, Faculty of Social Sciences, National Research University Higher School of Economics, Ph.D.

Research area: personality compensatory adaptive structures, mental resource, self-relation.

E-mail: skurginyan@hse.ru 